\title{
The MEGAFLOW Project - Numerical Flow Simulation for Aircraft
}

\author{
C.-C. Rossow, N. Kroll, and D. Schwamborn \\ 1 Deutsches Zentrum für Luft- und Raumfahrt e. V. (DLR) in the \\ Helmholtz-Association \\ 2 Institute of Aerodynamics and Flow Technology D-38108 Braunschweig, \\ Germany \\ cord.rossow@dlr.de, norbert.kroll@dlr.de
}

Summary. Some years ago the national CFD project MEGAFLOW was initiated in Germany, which combined many of the CFD development activities from DLR, universities and aircraft industry. Its goal was the development and validation of a dependable and efficient numerical tool for the aerodynamic simulation of complete aircraft which met the requirements of industrial implementations. The MEGAFLOW software system includes the block-structured Navier-Stokes code FLOWer and the unstructured Navier-Stokes code TAU. Both codes have reached a high level of maturity and they are intensively used by DLR and the German aerospace industry in the design process of new aircraft. Recently, the follow-on project MEGADESIGN was set up which focuses on the development and enhancement of efficient numerical methods for shape design and optimization. This paper highlights recent improvements and enhancements of the software. Its capability to predict viscous flows around complex industrial applications for transport aircraft design is demonstrated. First results concerning shape optimization are presented.

\section{Introduction}

Aerospace industry is increasingly relying on advanced numerical flow simulation tools in the early aircraft design phase. Today, computational fluid dynamics has matured to a point where it is widely accepted as an essential, complementary analysis tool to wind tunnel experiments and flight tests. Navier-Stokes methods have developed from specialized research techniques to practical engineering tools being used for a vast number of industrial problems on a routine basis [51]. Nevertheless, there is still a great need for improvement of numerical methods, because standards for simulation accuracy and efficiency are constantly rising in industrial applications. Moreover, it is crucial to reduce the response time for complex simulations, although the relevant geometries and underlying physical flow models are becoming increasingly complicated. In order to meet the requirements of German aircraft industry, the 
national project MEGAFLOW was initiated some years ago under the leadership of DLR [28, 29]. The main goal was to focus and direct development activities carried out in industry, DLR and universities towards industrial needs. The close collaboration between the partners led to the development and validation of a common aerodynamic simulation system providing both a structured and an unstructured prediction capability for complex applications. This software is still constantly updated to meet the requirements of industrial implementations.

In the first phase of the project the main emphasis was put on the improvement and enhancement of the block-structured grid generator MegaCads and the Navier-Stokes solver FLOWer. In a second phase the activities were focused on the development of the unstructured/hybrid Navier-Stokes solver TAU. Due to a comprehensive and cooperative validation effort and quality controlled software development processes both flow solvers have reached a high level of maturity and reliability. In addition to the MEGAFLOW initiative, considerable development and validation activities were carried out in several DLR internal and European projects which contributed to the enhancement of the flow solvers. The MEGAFLOW software is used in the German aeronautic industry and research organizations for a wide range of applications. Due to the use of common software, the process of transferring latest research and technology results into production codes has been considerably accelerated.

Recently, based on the MEGAFLOW network the national project MEGADESIGN (2004-2007) was set up [26]. Its main objective is to enhance and establish numerical shape optimization tools within industrial aircraft design processes. The project deals with several key issues including suitable techniques for geometry parameterization, meshing and mesh movement methods, efficiency and accuracy improvements of the flow solvers as well as flexible and efficient deterministic and stochastic based optimizers.

The present paper describes the features of the MEGAFLOW software and demonstrates its capability on the basis of several industrial relevant applications. Finally, the perspective and future requirements of CFD for industrial applications are shortly outlined.

\section{MEGAFLOW software}

The MEGAFLOW software offers flow prediction capabilities which are based on both block-structured and hybrid meshes. Details are given in [25].

\subsection{Grid Generation}

For the generation of block-structured grids the interactive system MegaCads has been developed. Specific features of the tool are the parametric construction of multi-block grids with arbitrary grid topology, generation of 
high-quality grids through advanced elliptic and parabolic grid generation techniques, construction of overlapping grids and batch functionality for efficient integration in an automatic optimization loop for aerodynamic shape design [12]. The limitation of MegaCads is the non automatic definition of the block topology which for rather complex configurations may result in a time consuming and labor intensive grid generation activity. Besides MegaCads, the commercial software package ICEM-HEXA and specialized in-house codes are used for specific applications.

In contrast to the block-structured approach, no major development activities have been devoted to the generation of unstructured meshes within the MEGAFLOW project. A strategic cooperation, however, has been established with the company CentaurSoft [3] which provides the hybrid grid generation package Centaur. The software consists of three major parts. An interactive program reads in the CAD data of the geometry under consideration, performs some CAD cleaning if necessary and sets up the grid generation process. In a second step the surface and volume grid are generated automatically. For viscous calculations a quasi-structured prismatic cell layer with a specified number of cells around the geometry surface ensures high resolution of boundary layer effects. In a third step grid adaptation may be used to locally refine grid resolution. During the cooperation the Centaur grid generation software has been substantially advanced for transport aircraft applications. Improvements are underway to include for example the generation of non isotropic elements and wake surfaces. Within the MEGADESIGN project the partner EADS-M is developing fully automatic hybrid grid generation software which is adapted to massively parallel distributed computers.

\subsection{Flow Solvers}

The main components of the MEGAFLOW software are the block-structured flow solver FLOWer and the unstructured hybrid flow solver TAU. Both codes solve the compressible three-dimensional Reynolds averaged Navier-Stokes equations for rigid bodies in arbitrary motion. The motion is taken into account by transformation of the governing equations. For the simulation of aero-elastic phenomena both codes have been extended to allow geometry and mesh deformation. In the following sections the specific features of the Navier-Stokes codes are briefly described.

\section{Block-Structured Navier-Stokes Code FLOWer}

The FLOWer-Code is based on a finite-volume formulation on block-structured meshes using either the cell vertex or the cell-centered approach. For the approximation of the convective fluxes a central discretization scheme combined with scalar or matrix artificial viscosity and several upwind discretization schemes are available [27]. Integration in time is performed using explicit 
multistage time-stepping schemes. For steady calculations convergence is accelerated by implicit residual smoothing, local time stepping and multigrid. Preconditioning is used for low speed flows. For time accurate calculations an implicit time integration according to the dual time stepping approach is employed. The code is highly optimized for vector computers. Parallel computations are based on MPI [6].

A variety of turbulence models is implemented in FLOWer, ranging from simple algebraic eddy viscosity models over one- and two-equation models up to differential Reynolds stress models. The Wilcox $\mathrm{k}-\omega$ model is the standard model in FLOWer which is used for all types of applications, while for transonic flow the linearized algebraic stress model LEA [42] and the nonlinear EARSM of Wallin [52] have shown to improve the prediction of shock locations. Furthermore, the SST model of Menter [36] is available for a better prediction of separating flows. All two-equation models can be combined with Kok's modification [23] for improved prediction of vortical flows. For supersonic flows different compressibility corrections are available. Recently, within the EU project FLOMANIA Reynolds stress models based on the Wilcox stress- $\omega$ model [53] and the so-called SSG/LRR- $\omega$ model, a combination of the Wilcox stress- $\omega$ and the Speziale-Sarkar-Gatski model [47], have been implemented into FLOWer [17]. Particularly the SSG/LRR- $\omega$ model has been applied to a wide variety of test cases, ranging from simple airfoils to complex aircraft configurations and from transonic to high-lift conditions. Generally improved predictions have been obtained, while the numerical behavior of the Reynolds stress models appeared to be as robust as that of two-equation models. Fig. 1 shows the predicted pressure and skin friction distribution obtained with the Wilcox k- $\omega$ and with the SSG/LRR- $\omega$ model for the Aerospatiale A airfoil at $M_{\infty}=0.15, \alpha=13.3^{\circ}, R e=2 \times 10^{6}$, demonstrating the improvement by Reynolds stress modeling.
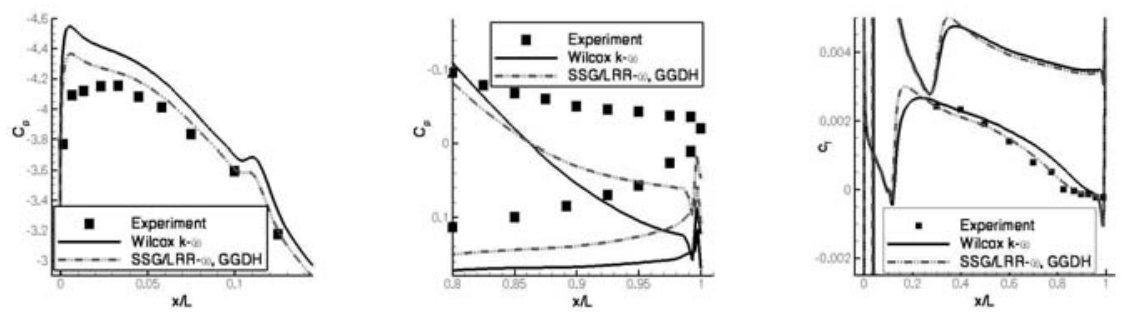

Fig. 1. Pressure distribution (near leading and trailing edge) and skin friction distribution for Aerospatiale A airfoil $\left(M_{\infty}=0.15, \alpha=13.3^{\circ}, R e=2 \times 10^{6}\right.$ ) calculated with the Reynolds stress turbulence model implemented in FLOWer.

Besides the modeling accuracy for turbulent flows, the numerical robustness of the respective transport equation turbulence models for complex ap- 
plications has been a major issue. In FLOWer numerical stability has been enhanced by an implicit treatment of the turbulence equations and different limiting mechanisms that can be activated by the user. The convergence behavior of the FLOWer-Code for a rather complex application is demonstrated in Fig. 2(a). Results of a viscous computation for a helicopter fuselage are shown [32]. The rotor is modeled through a uniform actuator disc. The grid consists of 94 blocks and 7 million grid points. The residuals for density and turbulence quantities are reduced several orders of magnitude. In this low Mach number case the preconditioning technique has been employed.
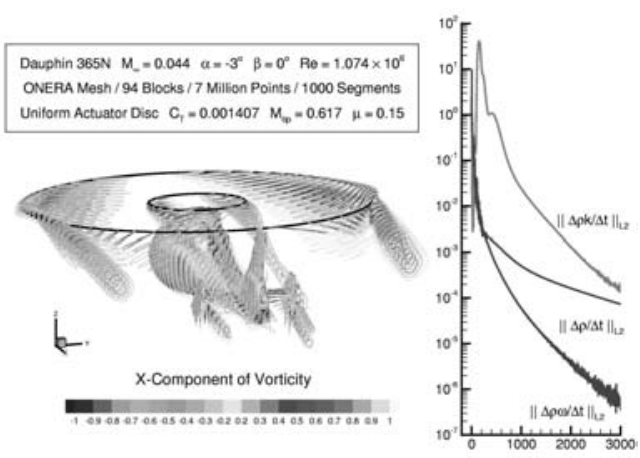

(a) Viscous calculation for Dauphin helicopter fuselage at $M_{\infty}=0.044$, convergence behavior of mass and $\mathrm{k}-\omega$ turbulence equations.

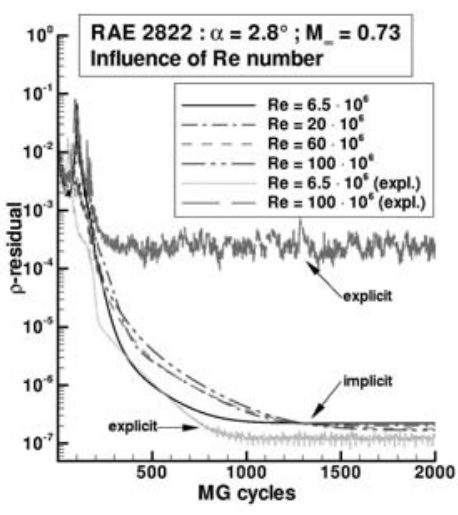

(b) Effect of Reynolds number on convergence for the RAE 2822 airfoil at $M_{\infty}=$ $0.73, \alpha=2.8^{\circ}$.

Fig. 2.

The fully implicit integration of the turbulence equations also ensures efficient calculations on highly stretched cells as they appear in high Reynolds number flows [18]. Fig. 2(b) shows the convergence history of FLOWer for the calculation of the viscous flow around the RAE 2822 airfoil at different Reynolds numbers. The advantage of the fully implicit method compared to the explicit multigrid scheme with point implicit treatment of source terms is evident.

FLOWer is able to perform transition prediction on airfoils and wings using a module consisting of a laminar boundary layer code and an $e^{N}$-database method based on linear stability theory [30]. Fig. 3 shows the predicted and measured force polars and transition locations of a subsonic laminar airfoil. This approach substantially improves the quality of predicted force coefficients. The experimentally determined transition points are reproduced with 
high accuracy. The transition prediction capability has been extended to $2 \mathrm{D}$ high-lift systems.

An important feature of FLOWer is the Chimera technique, which considerably enhances the flexibility of the block-structured approach [21, 45]. This technique mainly developed within the German/French helicopter project CHANCE [46] enables the generation of a grid around a complex configuration by decomposing the geometry into less complex components. Separate component grids are generated which overlap each other and which are em-

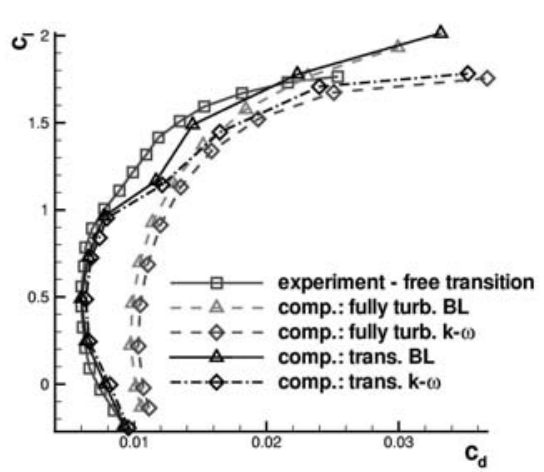

(a)

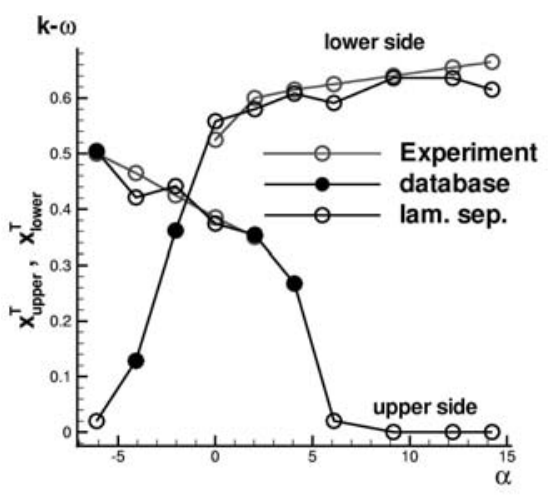

(b)

Fig. 3. Transition prediction with $e^{N}$-database method for laminar Sommers airfoil at $M_{\infty}=0.1$ and $R e=4 \times 10^{6}$, (a) force polars calculated fully turbulent and with transition, (b) computed and measured transition locations.

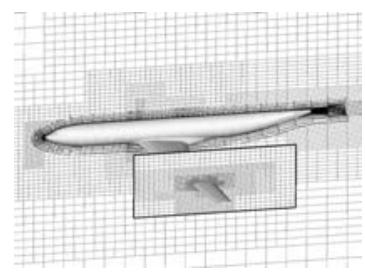

(a)

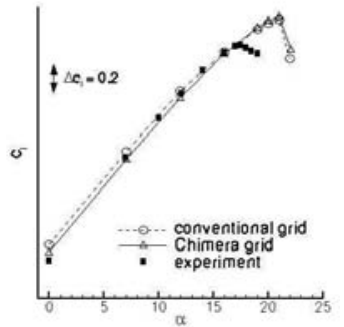

(b)

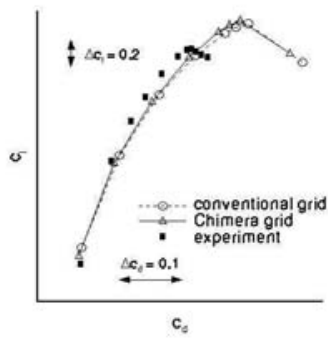

(c)

Fig. 4. Viscous computation about a $3 \mathrm{D}$ high-lift configuration using the Chimera technique of the block-structured FLOWer-Code, $M_{\infty}=0.174, \alpha=7^{\circ}$. 
bedded in a Cartesian background grid that covers the whole computational domain. In combination with flexible meshes, the Chimera technique enables an efficient way to simulate bodies in relative motion. The communication from mesh to mesh is realized through interpolation in the overlapping area. The search for cells which are used for interpolation is performed using an alternating digital tree method. In the case when a mesh overlaps a body which lies inside another mesh, hole cutting procedures have to be used in order to exclude the invalid points from computation. Further simplification of the grid generation procedure is achieved by a fully automatic Cartesian grid generator. The grid generator places fine grids around the component grids and puts successively coarsened grids around the fine grids. Patched grid interfaces with hanging nodes are used at the interface between the grid blocks of the Cartesian mesh. In the vicinity of the configuration the Cartesian grid generator creates non isotropic cells which are adapted to the size of the cells in the component grids. This ensures accuracy in the overlap regions. The potential of the Chimera technique is demonstrated in Fig. 4 in case of the viscous calculation around a $3 \mathrm{D}$ high-lift configuration. Separate component grids have been generated for body, wing, flap and slat. The background grid has been produced with the automatic Cartesian grid generator. With this approach the time for grid generation has been considerably reduced. The whole grid consists of 4 million points in total. Fig. 4(b) and Fig. 4(c) show the distribution of lift versus angle of attack and lift versus drag, respectively. The results obtained on the Chimera grid are compared with computations carried out on a conventional block-structured grid and with experimental data. It can be seen that the computations on the different meshes agree very well and they are in quite good correlation to the experiments. Differences between computations and experiments occur at the angle of attack where lift breaks down.

\section{Hybrid Navier-Stokes Code TAU}

The Navier-Stokes code TAU $[19,49]$ makes use of the advantages of unstructured grids. The mesh may consist of a combination of prismatic, pyramidal, tetrahedral and hexahedral cells and therefore combines the advantages of regular grids for the accurate resolution of viscous shear layers in the vicinity of walls with the flexibility of grid generation techniques for unstructured meshes. The use of a dual mesh makes the solver independent of the type of cells that the initial grid is composed of. Various spatial discretization schemes were implemented, including a central scheme with artificial dissipation and several upwind methods. The basic hybrid TAU-Code uses an explicit RungeKutta multistage scheme in combination with an explicit residual smoothing. In order to accelerate convergence, a multigrid procedure was developed based on the agglomeration of the control volumes of the dual grid for coarse grid computations. 
In order to efficiently resolve detailed flow features, a grid adaptation algorithm for hybrid meshes based on local grid refinement and wall-normal mesh movement in semi-structured near-wall layers was implemented. This algorithm has been extended to allow also for de-refinement of earlier refined elements thus enabling the code to be used for unsteady time-accurate adaptation in unsteady flows. Fig. 5 gives a simple example of the process for viscous airfoil calculation. First a flow solution is calculated on a basic grid (a). After some refinement an adapted grid/solution is obtained (b). Changing the flow parameters and specifying e.g. that the number of mesh points should not increase any further, the de-refinement interacts with the refinement (c) and finally the new shock position is resolved (d).

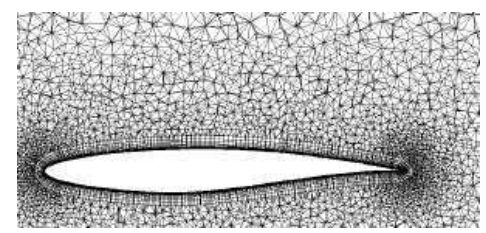

(a) initial state

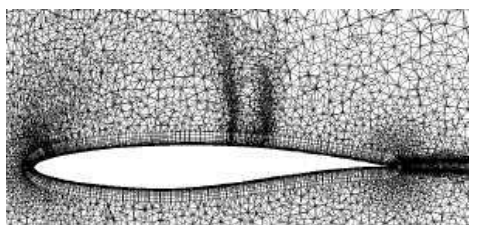

(c) intermediate state

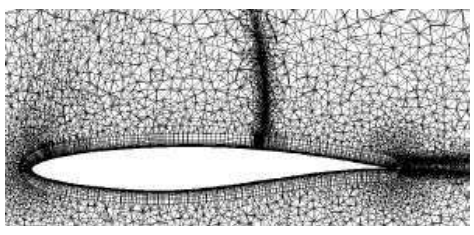

(b) adapted grif state 1

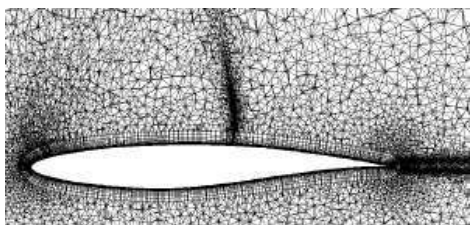

(d) sdapted grid state 2

Fig. 5. Demonstration of the dynamic mesh refinement and de-refinement capability of the TAU-Code.

With respect to unsteady calculations, the TAU-Code has been extended to simulate a rigid body in arbitrary motion and to allow grid deformation. In order to bypass the severe time-step restriction associated with explicit schemes, the implicit method based on the dual time stepping approach is used. For the calculation of low-speed flows, preconditioning of the compressible flow equations similar to the method used in FLOWer was implemented. One of the important features of the TAU-Code is its high efficiency on parallel computers. Parallelization is based on the message passing concept using the MPI library [6]. The code is further optimized either for cache or vector processors through specific edge coloring procedures. 
The standard turbulence model in TAU is the Spalart-Allmaras model with Edwards modification, yielding highly satisfactory results for a wide range of applications while being numerically robust. Besides this model, a number of different $\mathrm{k}-\omega$ models with and without compressibility corrections are available. Also nonlinear explicit algebraic Reynolds stress models (EARSM) and the linearized LEA model [42] have been integrated. Several rotation corrections for vortex dominated flows are available for the different models. Finally, there are options to perform detached eddy simulations (DES) based on the Spalart-Allmaras model [48] and so-called Extra-Large Eddy Simulations (XLES) [24].

The explicit character of the solution method severely restricts the CFL number which in turn often leads to slow convergence, especially in the case of large scale applications. In order to improve the performance and robustness of the TAU-Code, an approximately factored implicit scheme has been implemented [16]. The LU-SGS (Lower-Upper Symmetric Gauss-Seidel) scheme has been selected as a replacement for the Runge-Kutta scheme. In contrast to fully implicit schemes, this method has low memory requirements, low operation counts and can be parallelized with relative ease. Compared to the explicit Runge-Kutta method, the LU-SGS scheme is stable with almost no time step restrictions. An example of the performance improvement achieved is given in Fig. 6, where two convergence histories for viscous calculations on a delta wing are shown. The calculations were performed with multigrid on 16 processors of a Linux cluster. The figure shows the residual and the rolling moment against iteration count. In terms of iterations LU-SGS can be seen to converge approximately twice as fast as the Runge-Kutta scheme. Furthermore, one iteration of LU-SGS costs roughly $80 \%$ of one Runge-Kutta step. This results in a reduction of the overall calculation time by a factor of 2.5.
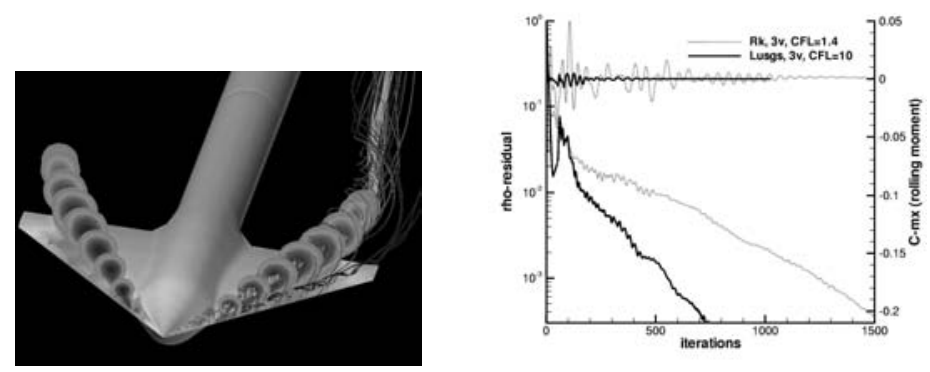

Fig. 6. Convergence behaviour of the hybrid TAU-Code for calculations of viscous flow around a delta wing at $M=0.5, \alpha=9^{\circ}$. Comparison of the baseline RungeKutta scheme (RK) and the implicit LU-SGS scheme.

As the Chimera technique has been recognized as an important feature to efficiently simulate maneuvering aircraft, it has been also integrated into 
the TAU-Code [34]. In the context of hybrid meshes the overlapping grid technique allows an efficient handling of complex configurations with movable control surfaces (see Fig. 7). For the intergrid communication linear interpolation based on a finite element approach is used in case of tetrahedral mesh elements. For other types of elements (prisms, hexahedrons, pyramids) linear interpolation is performed by splitting the elements into tetrahedrons. Like in FLOWer, the search algorithm for donor cells is based on the alternating digital tree data structure. The current implementation of the Chimera technique can handle both steady and unsteady simulations for inviscid and viscous flows with multiple moving bodies. The technique is available in parallel mode. In Fig. 8 results of a viscous Chimera calculation for a delta wing with trailing edge flaps are shown [43]. The component mesh of the flap is designed to allow a flap deflection of $\pm 15^{\circ}$. The comparison of calculated and measured surface pressure distributions at both $60 \%$ and $80 \%$ cord length shows good agreement.
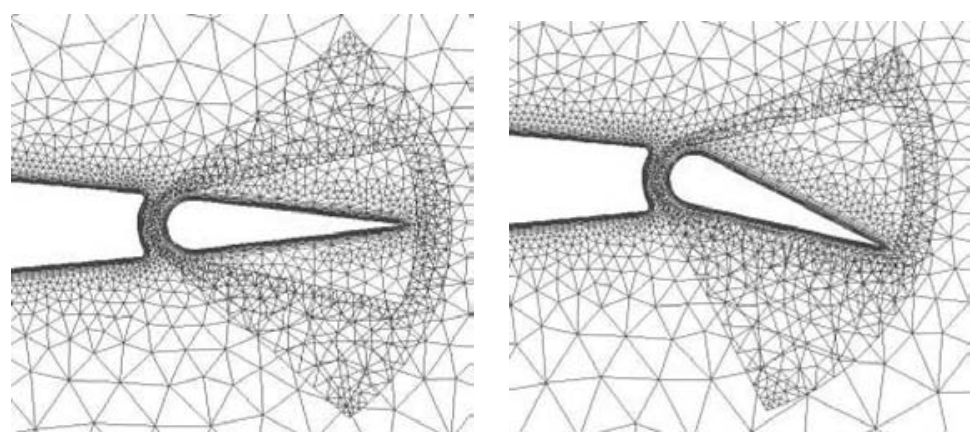

Fig. 7. Hybrid Chimera grid for delta wing with a movable flap.
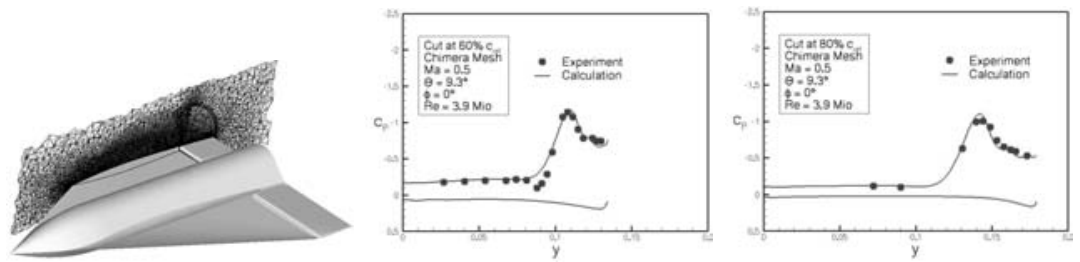

Fig. 8. Viscous computation of a delta wing with trailing edge flap using the Chimera option of the hybrid TAU-Code, surface pressure distributions for flap deflection angle $\theta=0^{\circ}$ at $60 \%$ and $80 \%$ cord. 


\section{Software validation}

Software validation is a central and critical issue when providing reliable CFD tools for industrial applications. Among others, the verification and validation exercises should address consistency of the numerical methods, accuracy assessment for different critical application cases and sensitivity studies with respect to numerical and physical parameters. Best practice documentation is an essential part of the work. Over the last few years the MEGAFLOW software has been validated within various national and international projects for a wide range of configurations and flow conditions (see e.g. [25, 40]). This section shows sample results for a subsonic and transonic validation test case.

Flow prediction for a transport aircraft in high-lift configuration is still a challenging problem for CFD. The numerical simulation addresses both complex geometries and complex physical phenomena. The flow around a wing with deployed high-lift devices at high incidence is characterized by the existence of areas with separated flow and strong wake/boundary layer interaction. The capabilities of the MEGAFLOW software to simulate two- and three-dimensional high-lift transport aircraft configurations has been extensively validated within the European high-lift program EUROLIFT I [39]. One of the investigated test cases is the DLR-F11 wing/body/flap/slat configuration.

Fig. 9 highlights a comparison of lift and total drag results of the unstructured TAU-Code and the block-structured FLOWer-Code with experimental data from the Airbus LWST low speed wind tunnel in Bremen, Germany. Both, the block-structured grid generated by the DLR software MegaCads and the hybrid mesh generated by FOI contain about 3 million grid points to allow for a fair comparison of the methods.
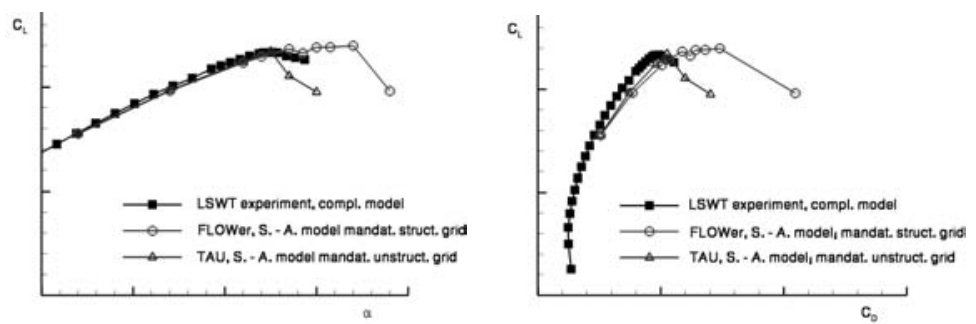

Fig. 9. Viscous computations for DLR-F11 high-lift configuration at $M_{\infty}=$ $0.18, R e=1.4 \times 10^{6}$, lift as function of angle of attack and as function of drag.

Calculations for the start configuration at $M_{\infty}=0.18$ and $R e=1.4 \times 10^{6}$ were performed with FLOWer and TAU using the Spalart-Allmaras turbulence model with Edwards modification (SAE). In both cases preconditioning was used to speed up steady state convergence and to improve accuracy at the predominantly low speed conditions. In the linear range of the polar, the 
numerical results compare quite well with each other and with experimental data. At higher angle of attack differences occur between the TAU and FLOWer results. TAU predicts the lift break down at a lower angle of attack, which is in better agreement with the experimental results.

In the framework of the AIAA CFD Drag Prediction Workshop I [1], the accuracy of the MEGAFLOW software was assessed to predict aerodynamic forces and moments for the DLR-F4 wing-body configuration [38]. In Fig. 10 lift coefficient as function of drag and angle of attack for Case $2\left(M_{\infty}=0.75\right.$, $R e=3 \times 10^{6}$ ) calculated with FLOWer and TAU are presented. These results were obtained using grids generated in-house at DLR. On request all calculations were performed fully turbulent. The FLOWer computations were carried out on a grid with 3.5 million points using central discretization with a mixed scalar and matrix dissipation operator and the $\mathrm{k} / \omega$-LEA turbulence model. The TAU results are based on an initial grid containing 1.7 million points which was adapted for each angle of attack yielding grids with 2.4 million points. In addition, an adaptation of the prismatic grid towards $y^{+}=1$ was done. Central discretization with standard settings of artificial dissipation was used. Turbulence was modeled with the one-equation model of Spalart-Allmaras. As can be seen from Fig. 10 the fully turbulent FLOWer computations over predict the measured drag curve by approximately 20 drag counts. Investigations have shown [38] that inclusion of transition in the calculation reduces the predicted drag by 14 drag counts, reducing the over prediction of drag to approximately 6 drag counts. The results of the unstructured fully-turbulent computations with TAU perfectly match with the experimental data. However, as for the structured computations, hybrid calculations with transition setting will reduce the predicted level of drag, in this case by approximately 10 drag-counts. Fig. 10 also shows the comparison of predicted and measured lift coefficient as a function of angle of attack. The values calculated by FLOWer agree very well with the experiment, whereas the results obtained with TAU over predict the lift almost in the whole range of angle of attack.

For the pitching moment (Fig. 11) the results obtained with FLOWer agree very well with experimental data. This is due to the fact that the surface pressure distribution predicted with the FLOWer-Code is in good agreement with the experiment. In case of the hybrid TAU-Code there are some discrepancies between the predicted and measured surface pressures resulting in a significant over prediction of the pitching moment. Further investigations [38] have shown that the improved results obtained with the FLOWer-Code are mainly attributed to a lower level of numerical dissipation (improved grid resolution and matrix dissipation) combined with the advanced 2-equation $\mathrm{k} / \omega$-LEA turbulence model.

Within the second AIAA drag prediction workshop [2] the hybrid TAUCode was further assessed with respect to performance calculations for a wing/body/pylon/nacelle configuration at transonic flow conditions [11]. For this exercise the Spalart-Allmaras one-equation turbulence model was used. 

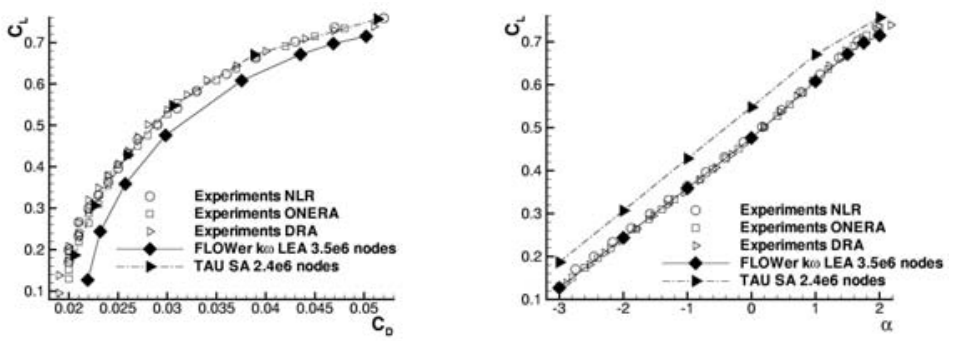

Fig. 10. Viscous calculations for DLR-F4 wing/body configuration (AIAA DPW I, case 2$), C_{L}\left(C_{D}\right), C_{L}(\alpha)$.
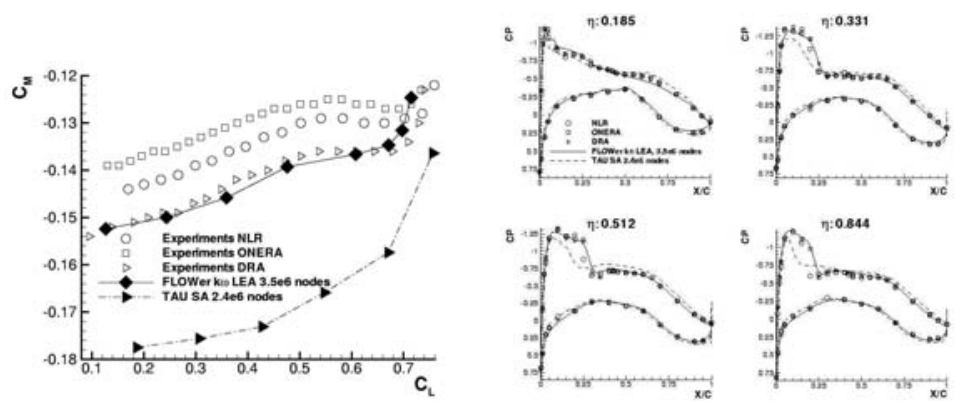

Fig. 11. Viscous calculations for DLR-F4 wing/body configuration (AIAA DPW I), $C_{M}\left(C_{L}\right)$ polar, surface pressure.
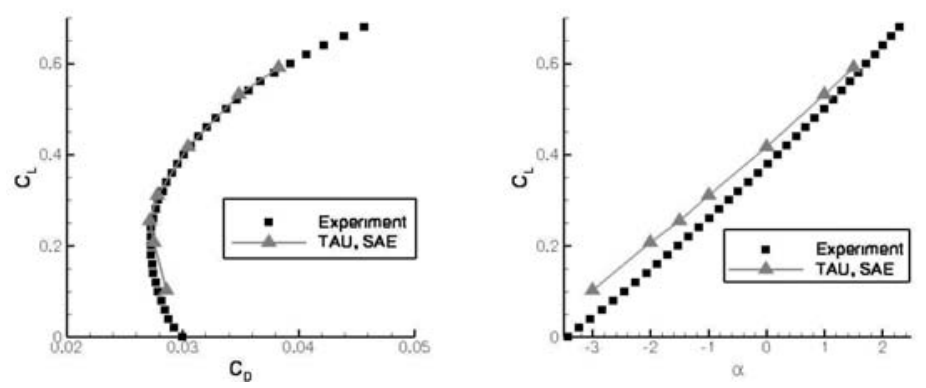

Fig. 12. TAU results for DLR-F6 wing/body/pylon/nacelle configuration (AIAA DPW II), $M_{\infty}=0.75$ 

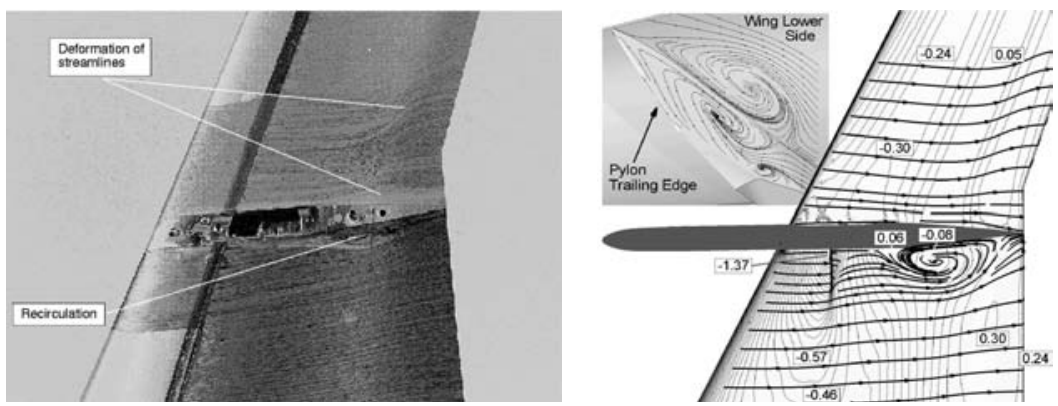

Fig. 13. Oil flow pictures (experiments) and streamlines (TAU results), DLR-F6 wing/body/pylon/nacelle configuration, wing lower and pylon inboard side, $M_{\infty}=$ $0.75, C_{L}=0.5$.

The drag polar is predicted in good agreement with the experimental data while the lift is constantly over predicted (see Fig. 12). A detailed analysis of the flow features reveals that in principle all areas of flow separations on the investigated DLR F6 configuration are identified, however, compared with experiments the sizes of those areas are slightly under predicted (wing upper side) or over predicted (wing lower side). Fig. 13 compares measured and predicted flow features near the pylon inboard side at the wing lower side. This difference results in systematic deviations of the pressure distributions and pitching moments.

\section{Industrial Applications}

The MEGAFLOW software is intensively used at DLR and the German aircraft industry for many aerodynamic problems. Some typical large scale applications listed below demonstrate the capability of the software to support aircraft and helicopter design.

\section{Civil transport aircraft at cruise conditions}

One key issue during the design of an enhanced civil aircraft is the efficient engine-airframe integration. Modern very high bypass ratio engines and the corresponding close coupling of engine and airframe may lead to substantial loss in lift and increased installation drag. At DLR, numerical and experimental studies have been devoted to estimate installation drag with respect to variations of engine concepts and the installation positions [13, 41]. For numerical investigations in this field both the block-structured FLOWer-Code and the hybrid TAU-Code have been used. Fig. 14 shows the hybrid grid in the symmetry plane for the DLR-F6 configuration [10]. The initial grid generated with Centaur consists of about 4.6 million nodes. Several solution based 
grid adaptation steps have been performed resulting in grids between 7.5 and 8.5 million nodes depending on the investigated engine concept. In Fig. 14 the lift as a function of the installation drag is plotted for three different positions of the CFM56 long duct nacelle $\left(M_{\infty}=0.75\right.$ and $\left.R e=3 \times 10^{6}\right)$. The engines are represented by through-flow nacelles. Results predicted with the TAU-Code (symbols) and measured in the ONERA S2MA wind tunnel (lines) are shown. The agreement is very satisfactory demonstrating that the influence on installation drag due to varying engines locations or sizes can be accurately predicted by the TAU-Code [10].
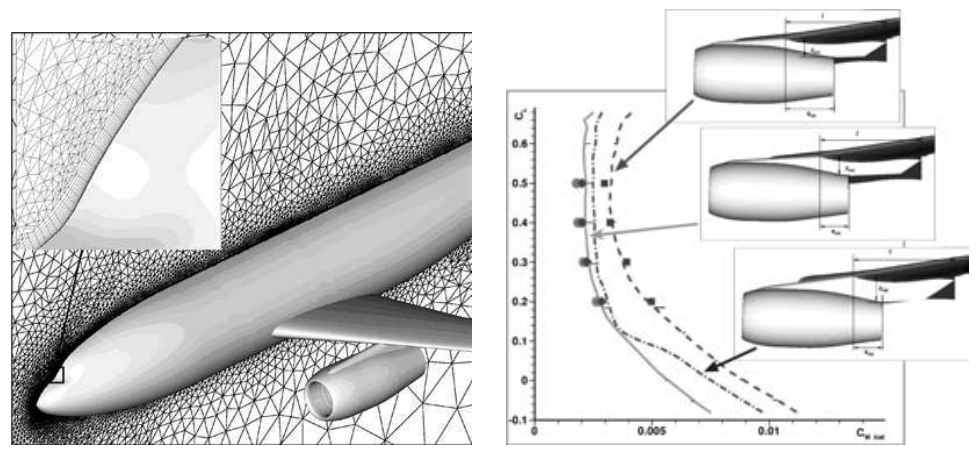

Fig. 14. Prediction of engine-airframe interference drag using the TAU-Code, left: hybrid grid for DLR-F6 configuration, right: lift as a function of installation drag for three different position of CFM56 engine, $M_{\infty}=0.75, R e=3 \times 10^{6}$, symbols: calculation, lines: experiment.

Viscous computations with the block-structured FLOWer-Code were performed for the DLR-ALVAST configuration with turbofan engines for the most interesting conditions 'Start of Cruise' (SOC), and 'Through Flow Nacelle' (TFN) representing a flight-idle power setting [41]. Computations were carried out at $M_{\infty}=0.75, R e=3 \times 10^{6}$ and with a constant lift coefficient of $C_{L}=0.5$. Fig. 15 shows the impact of the power setting. Computed lines of constant Mach number in the engine symmetry plane are shown. The primary differences caused by the SOC thrust condition are the strong velocity increase in the jets up to supersonic speed and the resulting significant shear layers at the jet boundaries due to the larger velocity differences. Fig. 15 also shows corresponding computed and measured pressure distributions at the wing cross section $\eta=33 \%$ (inboard of nacelle). The most significant difference between the SOC and TFN condition is a lower pressure level for SOC in the mid chord area at the wing lower side. This influence is captured quite well by the numerical simulation. 


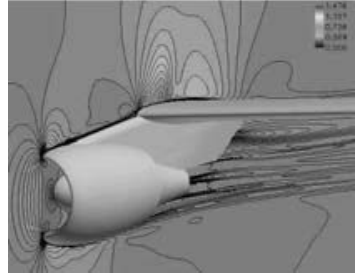

(a) $\mathrm{TFN}$

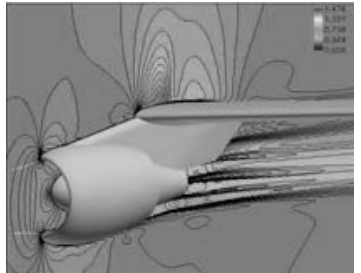

(b) SOC

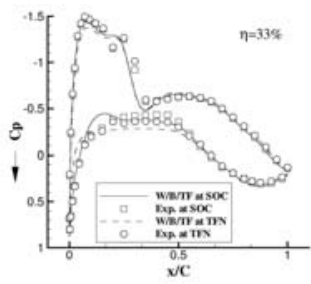

(c)

Fig. 15. Viscous calculation of DLR ALVAST configuration with FLOWer at $M_{\infty}=$ $0.75, C_{L}=0.5$, influence of thrust condition of turbofan engine, (a) and (b) constant Mach number distribution for TFN and SOC, (c) surface pressure distribution at cross section $\eta=33 \%$.

\section{Civil transport aircraft at high-lift conditions}

Based on thorough development and validation efforts of the hybrid unstructured approach employing both the Centaur grid generation software and the Navier-Stokes-Code TAU, complex high-lift flows become more and more accessible. As an example the flow around the DLR ALVAST model in high lift configuration equipped with two different engine concepts, the VHBR (Very High Bypass Ratio) and the UHBR (Ultra High Bypass Ratio) engine has been computed [35]. The numerical simulations are focused on complex flow phenomena arising from the engine installation at high-lift conditions. Special attention was paid to a possible reduction of the maximum lift angle resulting from dominant three-dimensional effects due to engine installation. Fig. 16 displays the surface pressure coefficient of the ALVAST high-lift configuration with installed VHBR and UHBR engine at an angle of attack of $\alpha=12^{\circ}$ in take-off conditions. The computations were performed on a hybrid grid with 10 million points generated by Centaur. In Fig. 17(a) the vortex shedding from the inboard side of the nacelle is shown. The vortex originates from the rolling-up of the shear layer and crosses the slat and the wing upper side. Using the computational data as input this vortex system could be identified with PIV visualization in a recent wind tunnel campaign. Fig. 17(a) also shows the impact of the two different engine concepts on the span wise lift distribution. For the VHBR concept the lift loss on the wing due to engine mounting is roughly compensated by the lift generated by the nacelle itself. For the UHBR concept the wing lift loss is slightly stronger than for the VHBR. Nevertheless, it is overcompensated by the higher lift carried by the large nacelle.

One key aspect of the development of a new transport aircraft is the design of a sophisticated and optimal high-lift system for take-off and landing conditions. A possibility to increase maximum lift is the usage of small delta wing like plates on the engine nacelles, the so-called nacelle strakes. These 
strakes generate vortices which run above the wing for high angles of attack. These vortices influence the wing and slat pressure distributions and shift the flow separations to higher angles of attack. At cruise flight conditions the strakes should not produce any significant additional drag. Previous investigations based on hybrid grid RANS solutions using the DLR TAU software have shown that for civil transport aircraft the influence of the nacelle strakes on lift and drag can be computed qualitatively [15]. In order to quantitatively predict the lift increment due to the strakes, care must be taken generating and adapting the grid with and without strakes. The idea has been to use the final adapted grid of the configuration with nacelle strakes and to fill the strakes with tetrahedral elements so that a nearly identical grid for the configuration with and without strakes can be build. The initial grid generation has been performed with Centaur. The element sizes have been controlled by several sources in the region where the strake vortices appear. The near wall region has been resolved by 25 layers of prismatic elements. The initial grid contains approximately 13.05 million points. The TAU grid adaptation has been used to insert additional points in areas of large gradients and to fulfill a $y^{+}$of nearly one. The three times adapted grid contains approximately 16.71 million points. The filling of the strake volume has been performed using customized tools based on MegaCads [12] and the NETGEN [4] software. Fig. 17(b) shows the adapted grid in the vicinity of the nacelle strake. The filled strake volume is visible. The solutions have been calculated using the TAU-Code for the flow condition $M_{\infty}=0.18, R e=3$ million and a between $8^{\circ}$ and $16^{\circ}$. Fig. 17 (c) demonstrates the resolution of the strake vortex and an iso-vorticity plane for $\alpha=10^{\circ}$. It has been shown that for this configuration a lift increase of $\Delta C_{L} \approx 0.1$ can be found both from the numerical calculations and the experiments although the absolute maximum lift values differ [14].
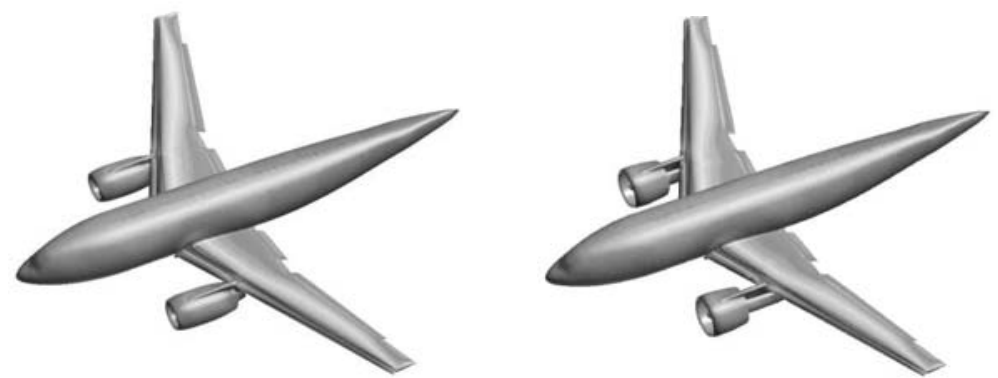

Fig. 16. Viscous simulation of the ALVAST high-lift configuration with VHBR (left) and UHBR (right) engine using the TAU-Code, surface pressure distribution, $M_{\infty}=0.22, \alpha=12^{\circ}, R e=2 \times 10^{6}$. 

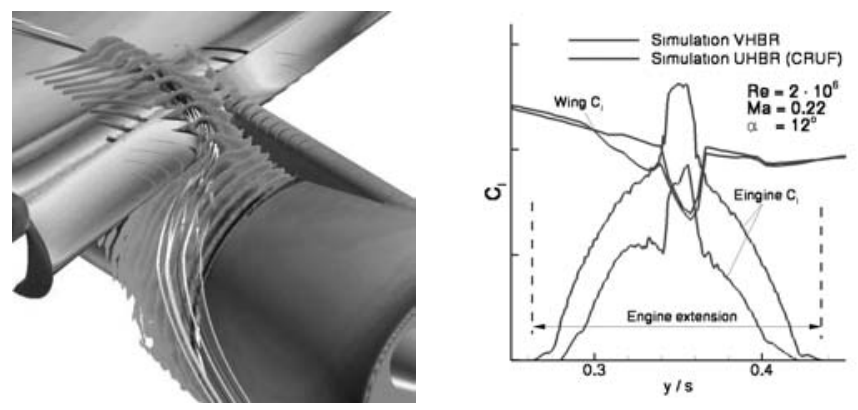

(a) Engine interference for ALVAST high-lift configuration with VHBR and UHBR engine $M_{\infty}=0.22, \alpha=$ $12^{\circ}, R e=2 \times 10^{6}$, left: nacelle vortex, right: lift distribution of wing and nacelle.
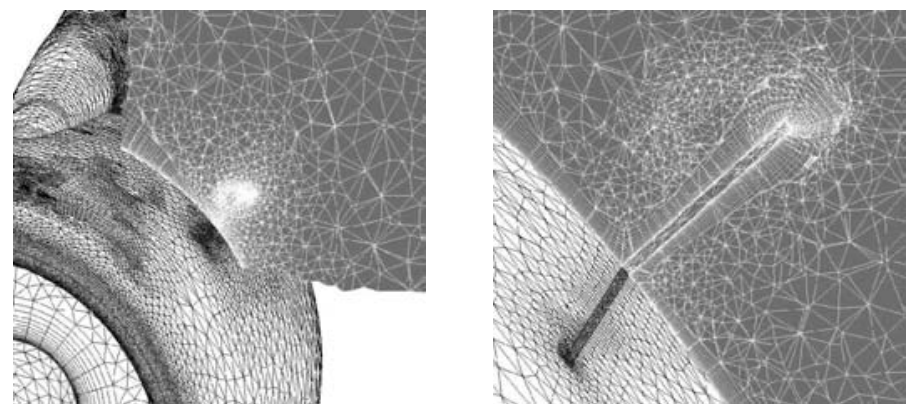

(b) Civil transport high-lift configuration with nacelle strakes, filled strake grid.
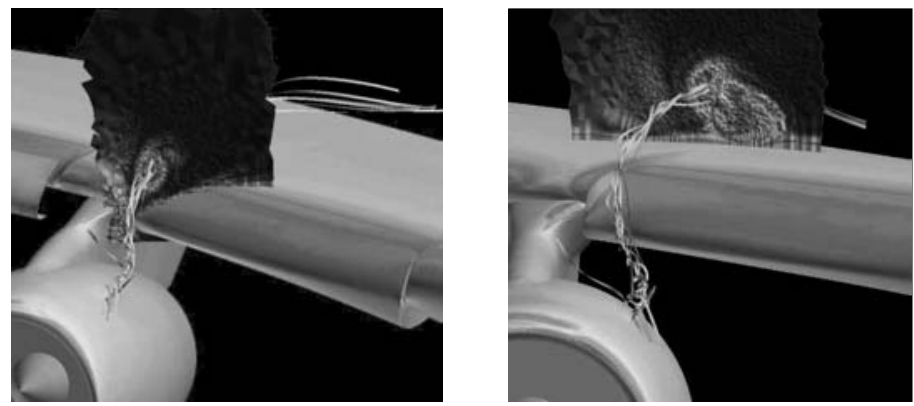

(c) Civil transport high-lift configuration with nacelle strakes, calculated streamlines and iso-vorticity cut planes.

Fig. 17. 


\section{Military aircraft}

Concerning military aircraft applications numerical simulations for the X-31 configuration have been carried out with the TAU-Code [5]. These computations show the capability of the TAU-Code to simulate complex delta wing configurations with rounded leading edges. Fig. 18(a) shows the numerically obtained 3D flow field over the X-31 configuration indicating the complexity of the vortex flow topology over the wing and the fuselage. Comparisons with experimental data show good agreement regarding the vortex topology. In Fig. 18(b) an oil flow picture of the X-31 clean wing from low speed experiments is shown in comparison to the corresponding CFD result. The angle of attack is $\alpha=18^{\circ}$ at a Reynolds number of 1.0 million. The attachment line of the strake vortex and the main wing vortex as well as the separation line of the main wing vortex near the leading edge is emphasized indicating that the flow topology from the calculation fits quite well with the experiment.

\section{Helicopter}

At DLR large effort is devoted to the enhancement of the MEGAFLOW software for helicopter applications. The development and validation activities are carried out in the German/French project CHANCE [46]. They include performance prediction of the isolated rotor in hover and forward flight as well as the quasi-steady and time-accurate simulation of the complete helicopter including engines and main and tail rotor.

The aerodynamic assessment of helicopter main rotors requires a computational procedure with fluid-structure coupling including trim. The results which are presented here were obtained with a weak coupling (see [37]) between the RANS solver FLOWer and the comprehensive rotor simulation code $\mathrm{S} 4$ in which the blade structure is modeled as a beam. The test case is the four-bladed 7A-rotor with rectangular blades in high-speed forward flight $\left(M_{\omega} R=0.64, M_{\infty}=0.256\right.$ with an advance ratio of $\left.\mu=0.4\right)$. Fig. $18(\mathrm{c})$ presents the grid system used while Fig. 18(d) compares the measured with the predicted data. The overall agreement of the coupled solution (FLOWer/S4 coupling) with the experimental data is acceptable although the negative peak in normal force around 120 azimuth is not well computed. This phenomenon is subject of ongoing research. The results of the simplified blade element aerodynamic module of S4 are presented by dashed lines in Fig. 18(d). It is obvious that this simplified aerodynamic model is not able to capture the time dependent blade load history.

A quasi-steady computation of the flow-field around the Eurocopter EC145 helicopter has been carried out $[32,31]$. The effect of engines and rotors has been simulated by means of in-/outflow boundary conditions and by actuator discs respectively. As visualized in Fig. 19(a), the rotor downwash results in an asymmetrical flow pattern on the fuselage surface. The figure shows separation lines and singular points on the boot and tail boom. Moreover, 


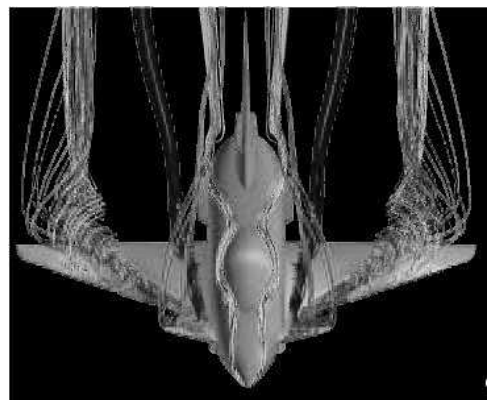

(a) 3D flow field of the X-31 configuration at $18^{\circ}$ angle of attack, TAU-Code.

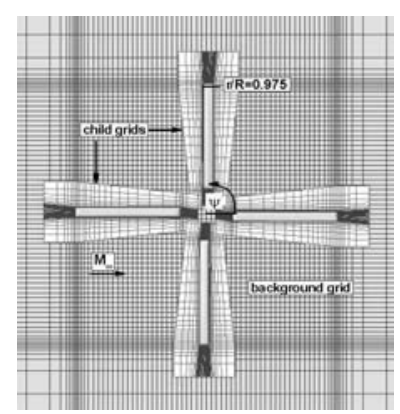

(c) Chimera grid system around 4-bladed 7A-rotor.

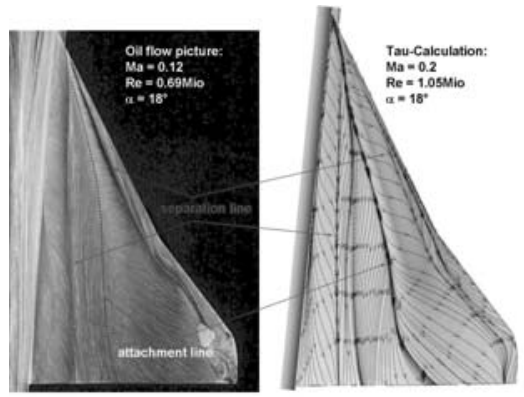

(b) X-31 clean wing, left: oil flow visualization, right: surface streamlines obtained with TAUCode.
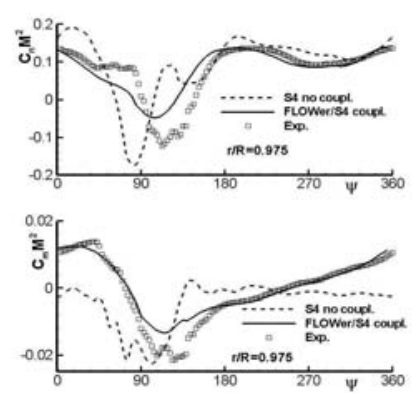

(d) Comparison of predicted and measured normal force and pitching moment coefficients versus azimuth for a high-speed forward flight test case of the $7 \mathrm{~A}$ rotor.

Fig. 18.

the right vertical stabilizer experiences a much higher loading as the left one. In Fig. 19(b) the surface temperature distribution and a 3D-contour for temperature of $T=60^{\circ} \mathrm{C}$ are depicted. Again the rotor downwash produces an asymmetrical temperature wake, which results in a single hot spot $\left(T=60^{\circ} \mathrm{C}\right)$ on the left horizontal stabilizer. 


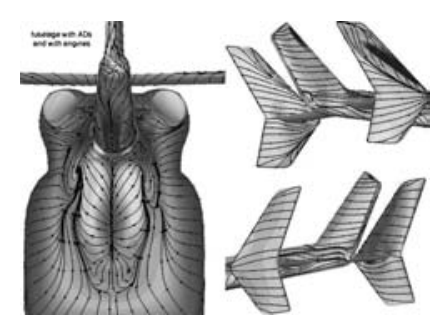

(a)

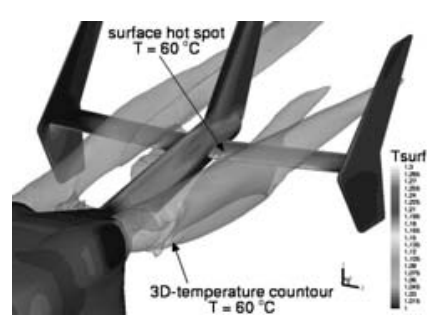

(b)

Fig. 19. (a) $C_{P}$-distribution and friction lines on the EC145 fuselage, visualisation of separation areas on the boot and vertical stabilisers. (b) Temperature surface distribution and $3 \mathrm{D}$-contour $\left(\mathrm{T}=60^{\circ} \mathrm{C}\right)$, visualisation of the impact of engine plumes on horizontal stabilisers.

\section{Multidisciplinary simulations}

The aerodynamic performance of large transport aircraft operating at transonic conditions is highly dependent on the deformation of their wings under aerodynamic loads. Hence accurate performance predictions require fluid/structure coupling in order to determine the aerodynamics of the configuration in aero-elastic equilibrium. Consequently, at DLR major effort is currently devoted to couple the flow solvers FLOWer and TAU with numerical methods simulating the structure. The activities include the development of efficient and robust grid deformation tools, accurate interpolation tools for transferring data between the fluid grid and the structure grid as well as the implementation of suitable interfaces between the flow solvers and the structural solvers. Concerning structure, both high-fidelity models (ANSYS, NASTRAN) and simplified models (beam model) are considered.

The importance of fluid/structure coupling is demonstrated in Fig. 20. Within the European project HiReTT Navier-Stokes calculations were performed for a wing-body configuration of a modern high speed transport type aircraft at $M_{\infty}=0.85$ and $R e=32.5 \times 10^{6}$. The block-structured FLOWerCode was used on a grid with about 3.5 million points. The $k / \omega$ turbulence model was employed. Two types of calculations were carried out. On the one hand the aerodynamic behavior of the jig-shape was predicted. On the other hand the aero-elastic equilibrium was determined by a fluid/structure coupling. For this calculation the coupling procedure of the University of Aachen (Lehr und Forschungsgebiet fr Mechanik) was used [8]. It is based on the FLOWer-Code for the fluid and a beam model for the structure. From Fig. 20 it is obvious that good agreement with experimental data obtained in the ETW can only be achieved with the fluid/structure coupling.

The improvement of maneuverability and agility is a substantial requirement of modern fighter aircraft. Most of today's and probably future fighter 
aircraft will be delta wing configurations. The flow field of such configurations is dominated by vortices resulting from flow separation at the wings and the fuselage. The time lag between vortex position and state with respect to the on-flow conditions of the maneuvering aircraft can lead to significant phase shifts in the distribution of loads. Reliable results for the analysis of the flight properties can only be achieved by a combined non-linear integration of the unsteady aerodynamics, the flight motion and the elastic deformation of the aircraft structure.

Within the DLR internal project SikMa [5, 44] a multidisciplinary simulation tool for maneuvering aircraft is being developed and validated. The unstructured, time-accurate flow solver TAU is coupled with a computational module solving the flight-mechanic equations and a structural mechanics code determining the structural deformations. By use of an overlapping grid technique (Chimera), simulations of complex configurations with movable control surfaces are possible. Fig. 21 shows an example of a multidisciplinary simulation of coupled aerodynamics and flight-mechanics. In this simulation the delta wing is released at a roll angle of zero degree and a pitching angle of $\alpha=9^{\circ}$ while the trailing edge flaps are deflected to $\eta= \pm 5^{\circ}$, respectively. On the upper right side of the figure the pressure distribution is shown at a stage where the flaps are fully deflected. On the upper left side the corresponding pitching and rolling moment are depicted as a function of the roll angle. The time histories of the rolling angle and the flap deflection angle are shown at the bottom of Fig. 21.
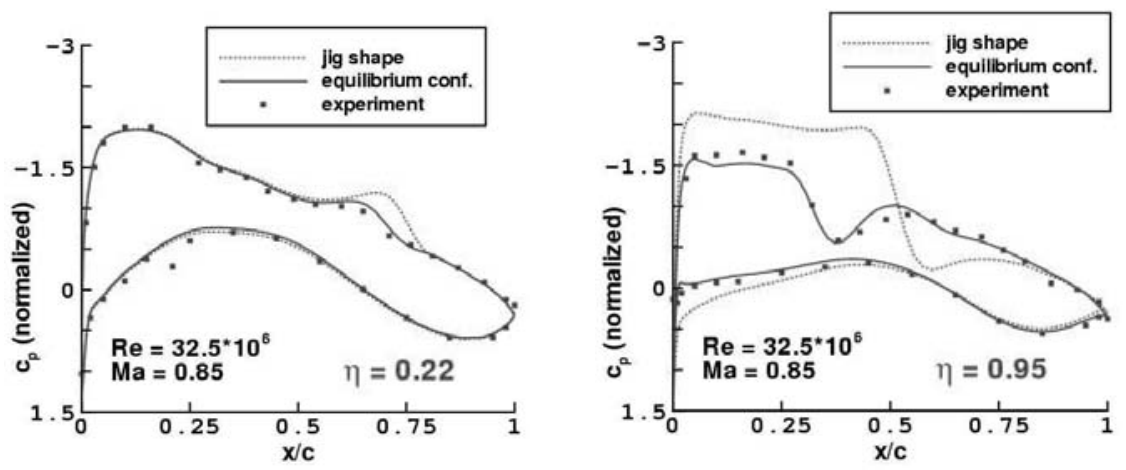

Fig. 20. $C_{P}$-distribution for different span wise sections for a wing/body configuration, numerical results obtained for pre-deformed geometry (dashed line) and with fluid/structure coupling (full line). 


\section{Numerical optimization}

For aerodynamic shape optimization, FLOWer and TAU offer an inverse design mode which is based on the inverse formulation of the small perturbation method according to Takanashi [50]. The method has been extended to transonic flows [7] and is capable of designing airfoils, wings and nacelles in inviscid and viscous flows.

In the context of regional aircraft development various wing designs for transonic flow were performed at DLR with the inverse mode of the NavierStokes solver FLOWer. As design target suitable surface pressure distributions were specified subject to geometrical constraints and a given lift coefficient. Fig. 22(a) shows the comparison of drag rise between an early baseline wing and an improved wing as a function of Mach number. The reduction of drag in the higher Mach number range is clearly visible. The constraint with respect to the lift coefficient was satisfied.

The inverse design methodology coupled to the hybrid TAU-Code was also applied to the design of wing-mounted engine nacelles [55]. Fig. 22(b) shows results of the redesign of an installed nacelle. The aircraft geometry under consideration is the DLR ALVAST wing/body/pylon/nacelle config-
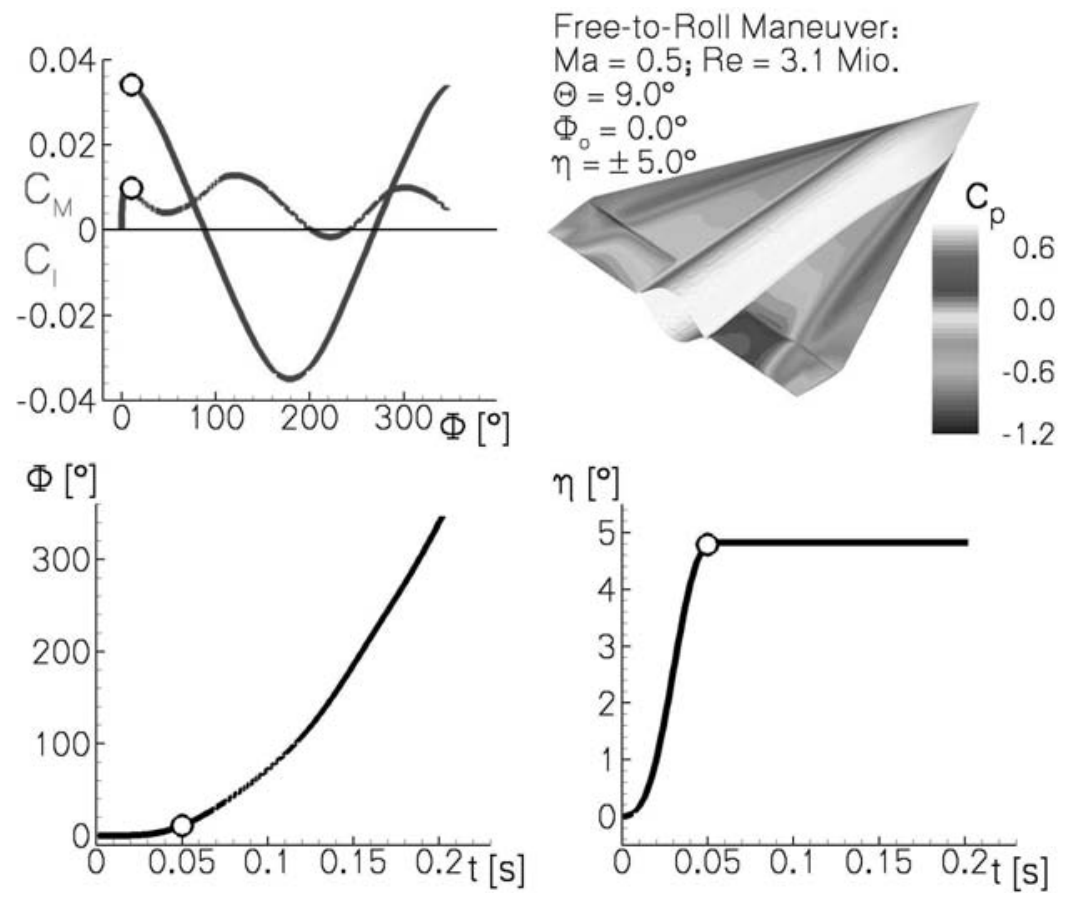

Fig. 21. Coupled aerodynamics and flight mechanics simulation for a rolling delta wing with trailing edge flaps using the TAU-Code. 
uration equipped with a VHBR engine. The initial nacelle geometry is set up by the scaled profiles of the side section only. The prescribed nacelle target pressure distribution corresponds to the surface pressure distribution of the installed VHBR nacelle. The redesign was performed for inviscid flow at $M_{\infty}=0.75, \alpha=1.15^{\circ}$ and the stream tube area ratio $\varepsilon_{F A N}=0.96$. Fig. $22(\mathrm{~b})$ shows surface pressure distributions and nacelle profiles in three circumferential sections. As can be seen, the prescribed pressure distributions are met in all three sections. This demonstrates that the inverse design methodology is capable of designing installed engine nacelles.

The inverse design method is very efficient; however it is restricted to a prescription of a target pressure distribution. A more general approach is the numerical optimization in which the shape, described by a set of design parameters, is determined by minimizing a suitable cost function subject to some constraints. At DLR high-lift system optimization is of major interest. Hence, the MEGAFLOW software has been coupled to various optimization strategies. As a demonstration results of a drag optimization for a 3-element airfoil in take-off configuration [54] are presented in Fig. 23. A limit in pitching moment has been prescribed as secondary constraint. In total 12 design variables are taken into account. These are slat and flap gap, overlap and deflection. In addition, the slat and flap cut-out contours are parameterized by three variables each. The optimization method is based on a deterministic SUBPLEX strategy. The Navier-Stokes FLOWer-Code is used to predict the flow field. The block-structured grid has about 80.000 grid points. In the left part of Fig. 23 the initial and optimized slat and flap contours are shown,

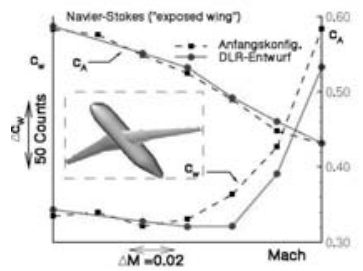

(a) Inverse wing design using FLOWer, drag rise lift as function of Mach number for baseline configuration and optimized configuration.

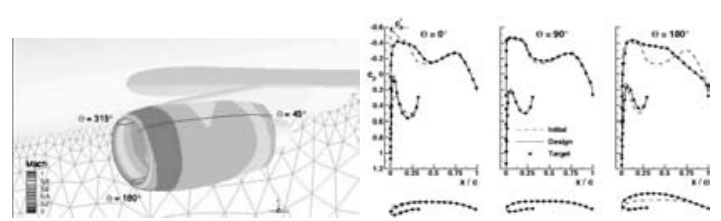

(b) Redesign of an installed nacelle using the TAU- Code, surface pressure distribution and nacelle profiles in three circumferential sections.

Fig. 22. 

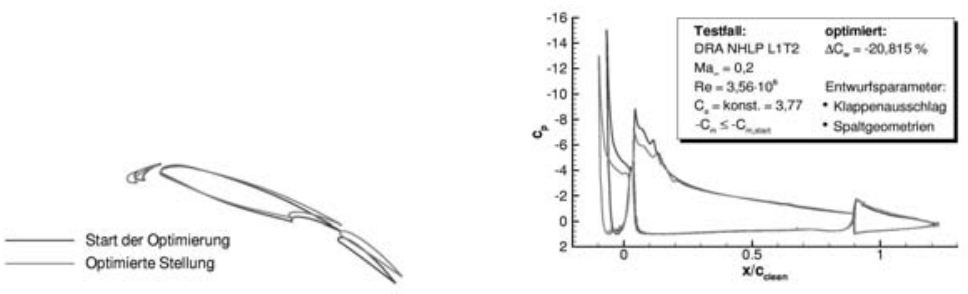

Fig. 23. Setting optimization of a 3-element airfoil using the FLOWer-Code.

in the right part the corresponding pressure distributions. The optimization affects the element chord, setting and deflection angle as well as the angle of attack. The optimization results in a decrease in total drag of $21 \%$, while the maximum lift is slightly improved by $2 \%$.

Because detailed aerodynamic shape optimizations still suffer from high computational costs, efficient optimization strategies are required. Regarding the deterministic methods, the adjoint approach is seen as a promising alternative to the classical finite difference approach (see e.g. [22]), since the computational cost does not depend on the number of design parameters. Accordingly, within the MEGAFLOW project an adjoint solver following the continuous adjoint formulation has been developed and widely validated for the block-structured flow solver FLOWer [20]. The adjoint solver can deal with the boundary conditions for drag, lift and pitching-moment sensitivities. The adjoint option of the FLOWer-Code has been validated for several $2 \mathrm{D}$ as well as 3D optimization problems controlled by the (adjoint) Euler equations. Within the ongoing MEGADESIGN project the robustness and efficiency of the adjoint solver will be further improved, especially for the Navier-Stokes equations. The adjoint solver implemented in FLOWer is currently transferred to the unstructured Navier-Stokes solver TAU.

To demonstrate the capability of the adjoint approach to handle many design parameters with low cost, the optimization of a supersonic transport wing/body configuration has been carried out [9]. The baseline geometry is based on the EUROSUP [33] geometry (Fig. 24), which is a supersonic commercial aircraft of 252 seats capacity, designed for a range of 5,500 nautical miles with supersonic cruise at Mach number $M_{\infty}=2.0$. The optimization goal is to minimize the drag at a fixed lift coefficient of $C_{L}=0.12$. The fuselage incidence is allowed to change in order to maintain the lift coefficient but it should not be greater than 4 degrees to the onset flow. In order to explore the full potential of the adjoint technique, no specific restrictions are set to define the parameterization. 74 design variables were used to change the twist, the thickness and the camber line at specific wing sections and 10 more design variables allowed changing the radial distribution of the fuselage. A minimum allowable value of the fuselage radius and a minimum wing thickness law were imposed in order to prevent unrealistic aircraft. After ge- 

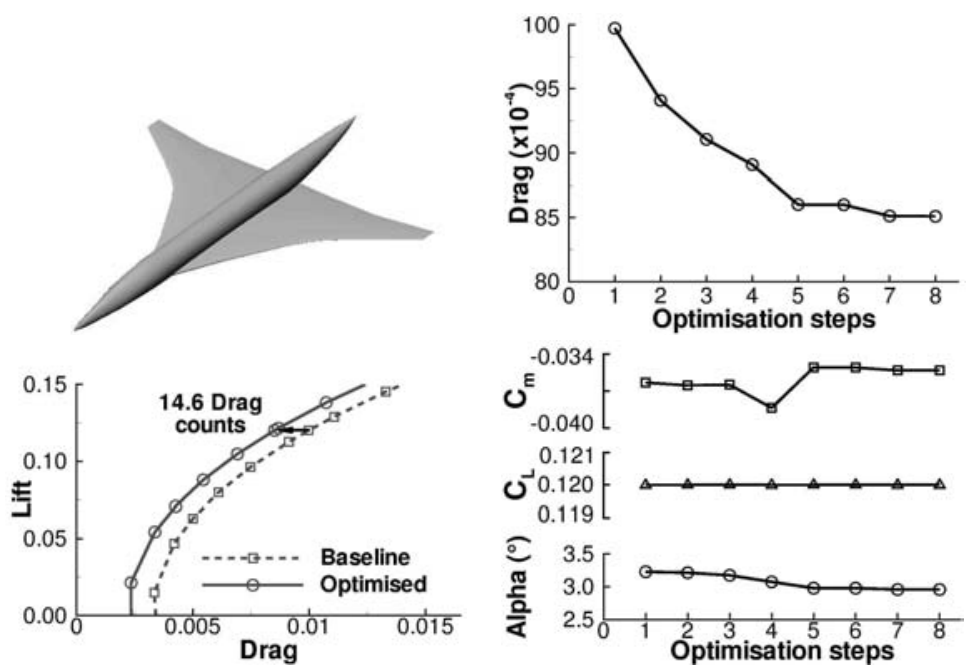

Fig. 24. Shape optimization of supersonic transport aircraft at $M_{\infty}=2.0$ (drag minimization at constant lift).

ometrical modifications, the intersection of wing and fuselage is recalculated automatically by the DLR in-house grid generator MegaCads for each new configuration. At $M_{\infty}=2.0$, the main aerodynamic effects are well predicted using the Euler equations. Therefore, the aerodynamic states are computed by FLOWer running in Euler mode. The constraint on the lift is handled using the target lift mode available in FLOWer which automatically adjusts the angle of attack to reach the desired lift. In the present optimization problem, the unique aerodynamic constraint is the lift, which is handled directly by FLOWer and the geometrical constraints are automatically fulfilled during the parameterization. Fig. 24 shows the evolution of the drag coefficient during the optimization, where an optimization step includes the evaluation of the gradient and the line search. About 8 optimization steps were necessary to achieve the optimum, which represents 54 aerodynamic computations and 8 adjoint flow evaluations. This approach is more than 11 times faster than using brute force optimization based on finite differences. The optimum configuration has 14.6 less drag counts than the baseline geometry. It can be seen in Fig. 24 that FLOWer keeps the lift constant during the complete optimization and the angle of attack decreases slightly by about 0.3 degrees. The pitching moment decreases by about $2.8 \%$. It is interesting to analyze the evolution of the performance around the design point. The lower left picture of Fig. 24 shows the polar both for the baseline and the improved geometries. It can clearly be seen that there is an almost constant reduction of the drag for the whole polar of the optimized geometry and not only at the main design point $\left(C_{L}=0.12\right)$. 


\section{Conclusions and perspective}

The main objective of the MEGAFLOW initiative was the development of a dependable, effective and quality controlled software package for the aerodynamic simulation of complete aircraft. Due to its high level of maturity, the MEGAFLOW software system is being used extensively throughout Germany for solving complex aerodynamic problems - especially in industrial development processes. However, since industry is still demanding more accurate and faster simulation tools, further development is required despite the high level of numerical flow simulation established today. Four major fields of further research activities may be identified:

The first field is the enhancement of numerical methods by new algorithms and solution strategies. Here, accuracy, robustness, and efficiency have to be addressed, while recognizing that these are contradicting requirements. In the design process of the aerospace industry with its severe time constraints, the difficult - with respect to required man-power usually unpredictable - set-up of highly accurate computations can not be tolerated. However, to establish numerical simulation during design, where decisions involving extreme economical risks have to be made, accuracy and reliability are crucial, which is why expensive wind tunnel testing is still indispensable. Furthermore, the efficiency of numerical methods has to be substantially improved. Relying solely on the progress of computational hardware is not an option, since over the last two decades the size of the problems to be simulated increased in parallel to or even faster than advancements in computer technology.

Second, the physical modeling of fluid flow needs further to be addressed. Despite long-time efforts, the current status of modeling of turbulence and transition is still inadequate for the highly complex flows to be simulated in aircraft design. Due to the immense computational effort required, the direct numerical simulation (DNS) or even Large Eddy Simulation (LES) of fluid flow will not be a practical alternative even for the next four or five decades. Therefore, reliable modeling of turbulence and transition will become decisive to bring numerical simulation as a routinely used tool into the aeronautical design process.

Third, the architecture of the simulation software is becoming more and more a strategic issue. On the one hand the software architecture must thoroughly exploit computational capabilities like parallelism, which requires a certain degree of dedication to a certain computational environment; on the other hand the software should be portable to different hardware arrangements. Furthermore, the software must be flexible with respect to coupling with other disciplines and integration into optimization strategies to allow the definition of an interdisciplinary simulation and optimization environment. At last, the software architecture must allow continuous upgrading for algorithmic and modeling improvements.

The last field to be addressed is validation. This requires on the one hand the thorough definition of suitable experiments by using most advanced mea- 
suring techniques. Especially for the envisaged simulation of unsteady flows with moving bodies and actuated control surfaces, corresponding experimental data are lacking. On the other hand, due to unavoidable effects such as grid dependency and limitations in physical modeling, the assessment of uncertainties in numerical simulation and a resulting statement of reliable applicability is becoming a major matter of future concern.

Development activities in the direction of the issues summarized above have been initiated in the now ongoing German CFD project MEGADESIGN, which is a follow-on project to the German MEGAFLOW initiative.

Acknowledgement. The authors would like to thank their colleagues of the DLR Institute of Aerodynamics and Flow Technology for providing the material presented in this paper. Thanks also to C. Braun from the University of Aachen, who provided the numerical results shown in Figure 27. Furthermore, the partial funding of the MEGAFLOW and MEGADESIGN project through the German Government in the framework of the aeronautical research program is gratefully acknowledged.

\section{References}

1. $1^{\text {st }}$ AIAA CFD Drag Prediction Workshop. http://www.aiaa.org/tc/apa/dragpredworkshop/dpw.html.

2. $2^{\text {nd }}$ AIAA CFD Drag Prediction Workshop. http://ad-www.larc.nasa.gov/tsab/cfdlarc/aiaa-dpw/.

3. CentaurSoft: http://www.centaursoft.com/.

4. NETGEN, http://www.hpfem.jku.at/netgen, 2004.

5. Schütte. A., G. Einarsson, B. Schöning, A. Raichle, W. Mönnich, Th. Alrutz, Neumann. J., and J. Heinnecke. Numerical Simulation of Maneuvering Combat Aircraft. In STAB 2004, to appear in Notes on Numerical Fluid Mechanics and Multidisciplinary Design (NNFM). Springer Verlag, 2005.

6. P. Aumann, H. Barnewitz, H. Schwarten, K. Becker, R. Heinrich, B. Roll, M. Galle, N. Kroll, Th. Gerhold, and M. Schwamborn, D.and Franke. MEGAFLOW: Parallel Complete Aircraft CFD. Parallel Computing, 27:415440, 2001.

7. W. Bartelheimer. An Improved Integral Equation Method for the Design of Transonic Airfoils and Wings. In AIAA 95-1688, 1995.

8. C. Braun, A. Bouche, and J. Ballmann. Numerical Study of the Influence of Dynamic Pressure and Deflected Ailerons on the Deformation of a High Speed Wing Model. In Krause. E., W. Jaeger, and M. Resch, editors, High Performance Computing in Science and Engineering 2004. Springer Verlag, 2004.

9. J. Brezillon and N.R. Gauger. 2D and 3D Aerodynamic Shape Optimization Using Adjoint Approach. Aerosp. Sci. Technol., 8:715-727, 2004.

10. O. Brodersen. Drag Prediction of Engine-Airframe Interference Effects Using Unstructured Navier-Stokes Calculations. Journal of Aircraft, 39(6):927-935, 2002.

11. O. Brodersen, M. Rakowitz, S. Amant, Larrieu. P., D. Destarac, and M. Sutcliffe. Airbus, ONERA and DLR Results from the 2nd AIAA Drag Prediction Workshop. In AIAA 2004-0391, 2004. 
12. O. Brodersen, A. Ronzheimer, R. Ziegler, T. Kunert, J. Wild, and M. Hepperle. Aerodynamic Applications Using Megacads. In M. Cross et al., editor, $6^{\text {th }}$ International Conference on Numerical Grid Generation on Computational Field Simulations, ISGG, pages 793-802, 1998.

13. O. Brodersen and A. Stürmer. Drag Prediction of Engine-Airframe Interference Effects Using Unstructured Nvier-Stokes Calculations. In AIAA 2001-2414, 2001.

14. O. Brodersen and J. Wild. DLR-IB 124-2004-18, 2004.

15. O. Brodersen, J. Wild, S. Melber-Wilkending, and L. Lekemark. In DLR-IB 129-2003-34, DLR Braunschweig, 2003.

16. R. Dwight. A Comparison of Implicit Algorithms for the Navier-Stokes Equations on Unstructured Grids. In Proceedings of the ICCFD Conference, Toronto, Canada, 2004.

17. B. Eisfeld. Numerical Simulation of Aerodynamic Problems with a Reynolds Stress Turbulence Model. In STAB 2004, to appear in Notes on Numerical Fluid Mechanics and Multidisciplinary Design (NNFM). Springer Verlag, 2005.

18. J.K. Fassbender. Improved Robustness for Numerical Simulation of Turbulent Flows around Civil Transport Aircraft at Flight Reynolds Numbers. In $D L R-F B$ 2003-09, 2003.

19. M. Galle. Ein Verfahren zur numerischen Simulation kompressibler, reibungsbehafteter strömungen auf hybriden netzen. In DLR-FB 99-04, 1999.

20. N.R. Gauger. Das Adjungiertenverfahren in der aerodynamischen Formoptimierung. In DLR-FB 2003-05, 2003.

21. R. Heinrich and N. Kalitzin. Numerical Simulation of Three-Dimensional Flows Using the Chimera Technique. Notes on Numerical Fluid Mechanics, 72:15-23, 1999.

22. A. Jameson, L. Martinelli, and N. Pierce. Optimum Aerodynamic Design Using the Navier-Stokes Equations. Theoret. Comput. Fluid Dynamics, 10:213-237, 1998.

23. J.C. Kok and F.J. Brandsma. Turbulence Model Based Vortical Flow Computations for a Sharp Edged Delta Wing in Transonic Flow Using the Full Navier-Stokes Equations. In NLR-CR-2000-342, 2000.

24. J.C. Kok, H.S. Dol, B. Oskam, and H. van der Ven. Extra-Large Eddy Simulation of Massively Separated Flows. In AIAA-Paper, 2004-0264, 2004.

25. N. Kroll and J.K. Fassbender(Eds.). Notes on Numerical Fluid Mechanics and Multidisciplinary Design, volume 89, chapter MEGAFLOW - Numerical Flow Simulation for Aircraft Design. Springer, 2005.

26. N. Kroll, Gauger. N. R., J. Brezillon, K. Becker, and V. Schulz. Ongoing Activities in Shape Optimization within the German Project MEGADESIGN. In ECCOMAS 2004, Jyväskylä, Finland, July 2004.

27. N. Kroll, R. Radespiel, and C.-C. Rossow. Accurate and Efficient Flow Solvers for 3D-Applications on Structured Meshes. In AGARD R-80\%, pages 4.1-4.59, 1995.

28. N. Kroll, C.C. Rossow, K. Becker, and F. Thiele. The MEGAFLOW project. Aerosp. Sci. Technol., 4:223-237, 2000.

29. N. Kroll, C.C. Rossow, D. Schwamborn, K. Becker, and G. Heller. MEGAFLOW - A Numerical Flow Simulation Tool for Transport Aircraft Design. ICAS, $1.10 .5,2002$. 
30. A. Krumbein. Coupling of the DLR Navier-Stokes Solver FLOWer with an $\mathrm{e}^{N}$-Database Method for Laminar-Turbulent Transition Prediction on Airfoils. Notes on Numerical Fluid Mechanics, 77:92-99, 2002.

31. F. Le Chuiton. Chimera simulation of a complete helicopter with rotors as actuator discs. In STAB 2004, to appear in Notes on Numerical Fluid Mechanics and Multidisciplinary Design (NNFM). Springer Verlag, 2005.

32. F. LeChuiton. Actuator Disc Modeling for Helicopter Rotors. Aerosp. Sci. Technol, 8:285-297, 2004.

33. D.A. Lovell. Aerodynamic Research to Support a Second Generation Supersonic Transport Aircraft - the EUROSUP Project. In ECCOMAS 1998, 1998.

34. A. Madrane, A. Raichle, and A. Stürmer. Parallel Implementation of a Dynamic Overset Unstructured Grid Approach. In ECCOMAS 2004, Jyväskylä, Finland, July 2004.

35. S. Melber. 3D RANS Simulations for High-Lift Transport Aircraft Configurations with Engines. DLR-IB 124-2002/27, 2002.

36. F.R. Menter. Two-Equation Eddy-Viscosity Turbulence Models for Engineering Applications. AIAA Journal, 32:1598-1605, 1994.

37. K. Pahlke and B. van der Wall. Chimera Simulations of Multibladed Rotors in High-Speed Forward Flight with Weak Fluid-Structure-Coupling. In 29th European Rotorcraft Forum, page 63, Friedrichshafen, Germany, 2003.

38. M. Rakowitz, M. Sutcliffe, B. Eisfeld, D. Schwamborn, and J. Bleecke, H.and Fassbender. Structured and Unstructured Computations on the DLR-F4 Wing-Body Configuration. In AIAA 2002-0837, 2002.

39. R. Rudnik. Towards CFD Validation for 3D High Lift Flows - EUROLIFT. In ECCOMAS 2001, Swansea, United Kingdom, 2001.

40. R. Rudnik, S. Melber, A. Ronzheimer, and O. Brodersen. Three-Dimensional Navier-Stokes Simulations for Transport Aircraft High Lift Configurations. Journal of Aircraft, 38:895-903, 2001.

41. R. Rudnik, C.C. Rossow, and H. v. Geyr. Numerical Simulation of Engine/Airframe Integration for High-Bypass Engines. Aerosp. Sci. and Technol., 6:31-42, 2002.

42. T. Rung, H. Lübcke, M. Franke, L. Xue, F. Thiele, and S. Fu. Assessment of Explicit Algebraic Stress Models in Transonic Flows. In Proceedings of the $4^{t} h$ Symposium on Engineering Turbulence Modeling and Measurements, pages 659-668, France, 1999.

43. A. Schütte, G. Einarsson, A. Madrane, B. Schöning, W. Mönnich, and W.-R. Krüger. Numerical Simulation of Maneuvering Aircraft by CFD and Flight Mechanic coupling. In RTO Symposium, Paris, April 2002.

44. A. Schütte, G. Einarsson, B. Schöning, A. Madrane, W. Mönnich, and W. Krüger. Numerical Simulation of Manoeuvring Aircraft by Aerodynamic and Flight Mechanic Coupling. In RTO AVT Symposium Paris, 2005.

45. Th. Schwarz. Development of a Wall Treatment for Navier-Stokes Computations Using the Overset Grid Technique. In 26th European Rotorcraft Forum, page 45, 2000 .

46. J. Sidès, K. Pahlke, and M. Costes. Numerical Simulation of Flows around Helicopters at DLR and ONERA. Aerosp. Sci. Technol., 5:35-53, 2001.

47. C.G. Speziale, S. Sarkar, and T.B. Gatski. Modeling the pressure-strain correlation of turbulence: an invariant dynamical systems approach. Journal of Fluid Mechanics, 227:245-272, 1991. 
48. M. Strelets. Detached Eddy Simulation of massively separated flows. In AIAAPaper 2001-0879, 2001.

49. Gerhold T., O. Friedrich, Evans J., and M. Galle. Calculation of Complex ThreeDimensional Configurations Employing the DLR-TAU Code. In AIAA 97-0167, 1997.

50. S. Takanashi. Iterative Three-Dimensional Transonic Wing Design Using Integral Equations. Journal of Aircraft, 22(8), 1985.

51. J.B. Vos, A. W. Rizzi, D. Darracq, and E. H. Hirschel. Navier-Stokes Solvers in European Aircraft Industry. Progress in Aerospace Sciences, 38:601-697, 2002.

52. S. Wallin and A.V. Johansson. An Explicit Algebraic Reynolds Stress Model for Incompressible and Compressible Turbulent Flows. J. Fluid Mech., 403:89-132, 2000 .

53. D.C. Wilcox. Turbulence Modeling for CFD, DCW Industries. CA, La Cañada, 1998.

54. J. Wild. Validation of Numerical Optimization of High-Lift Multi-Element Airfoils based on Navier-Stokes-Equations. In AIAA 2002-2939, 2002.

55. R. Wilhelm. An Inverse Design Method for Designing Isolated and WingMounted Engine Nacelles. In AIAA 2002-0104. 